\title{
Status of robotic-assisted surgery among Canadian urology residents
}

\author{
Michael Robinson, MD; Andrew MacNeily, MD, FRCSC, FAAP; Larry Goldenberg, CM, OBC, MD, FRCSC; \\ Peter Black, MD, FRCSC, FACS
}

Department of Urologic Sciences, Faculty of Medicine, University of British Columbia, Vancouver, BC

Cite as: Can Urol Assoc J 2012;6(3):160-7. htrp://dx.doi.org/10.5489/cuaj.11190

\section{Abstract}

Background: Robotic-assisted surgery (RAS) has been rapidly adopted in urology, especially in the United States. Although less prevalent in Canada, RAS is a growing and controversial field that has implications for resident training. We report on the status and perception of RAS among Canadian urology residents.

Methods: All Canadian urology residents from anglophone programs were contacted by email and asked to participate in an online survey. Current resident exposure to, and perception of, RAS was assessed.

Results: Of the residents contacted ( $\mathrm{n}=128), 50$ (39\%) completed the survey. Of the respondents, 52\% have been involved in RAS. Those who have not been involved in RAS express lower interest and lesser knowledge of RAS. Ninety-two percent of respondents feel the use of RAS will increase, although only $29 \%$ feel this is feasible in Canada. Just $24 \%$ and $36 \%$ feel RAS to be superior to open and laparoscopic techniques, respectively. Sixty-eight percent of residents in programs with a robot viewed it as detrimental to training, whereas $81 \%$ of residents in programs without one viewed its absence to either have no impact, or even be beneficial. Both groups expressed a desire for more experience with RAS.

Conclusion: The resident experience with respect to RAS is mixed. Overall, residents view RAS as an expanding field with potentially negative impacts on their present training, although they appear to desire the acquisition of more experience in RAS. We plan to monitor the evolution of these perceptions over next four years.

\section{Introduction}

Robotics were first applied in surgery during the mid-1980s; in 1989, the first urologic procedure, a robotic-assisted transurethral resection of the prostate, was performed. ${ }^{1,2}$ Since that time, robotic-assisted surgery (RAS) has grown exponentially as surgeons have looked to decrease operative and long-term morbidity, while adhering to the stringent principles of oncologic treatment. While RAS is considered superior in many circumstances to either traditional open or laparoscopic techniques, high-level evidence to support this contention is lacking. ${ }^{3,4}$ Notwithstanding the controversy, RAS has been rapidly adopted in urology, especially in the United States. Although less prevalent in Canada, the growth of RAS has implications for a number of areas in medicine, especially in resident training.

Attitudes among residents towards RAS differ across surgical disciplines. ${ }^{5}$ Resident perception is a key component of resident satisfaction, with consequences for recruitment and training. ${ }^{6-8}$ Such consequences may also have implications on faculty satisfaction. ${ }^{9}$ Furthermore, residents and staff are known to have different views regarding resident learning needs. ${ }^{6,8,10}$ Given all of this, one must investigate the urology resident pool directly for an accurate understanding of how RAS affects urologic training in Canada. Only with this understanding can we optimize our learning environments and curricula, attract the best and brightest individuals to our specialty, and develop surgeons of the highest calibre.

\section{Methods}

All 128 Canadian urology residents in postgraduate years one through five at each of the 10 anglophone programs were contacted by email and asked to participate in an online survey. Responses were collected over a four-week period beginning in February 2010. Of the 10 anglophone urology programs in Canada, there are five with nine da Vinci Surgical Systems (Intuitive Surgical, Inc., Sunnyvale, $\mathrm{CA})$; the other five programs do not have the robot. Residents were asked to complete the survey regardless of whether their program possessed a robot. Details of the study and consent were clearly described in the invitation email and residents who agreed to participate followed a link to the survey hosted on a secure online database. The survey (Appendix 1, Appendix 2) was designed to determine current resident exposure and perception towards RAS. For the purpose of this study, RAS was strictly defined to include only experiences with the da Vinci Surgical System. Survey 
participation was anonymous and no identifying information was collected. The survey was available for completion over a four-week period. A reminder was sent to all residents two weeks following the initial contact.

\section{Results}

Of the residents contacted $(n=128), 50(39 \%)$ responded. The highest response rate from three similar surveys performed at American institutions was 35\%. ${ }^{10-12}$ We tallied respondent demographics (Table 1). The proportion of Canadian urology residents with access to a robot in their program is $59 \%$, compared to $41 \%$ who do not. This statistic closely mirrors the respective proportion of responses from residents in this survey $(64 \%$ from programs with a robot and $36 \%$ from programs with no robot). In terms of experience with RAS, $52 \%$ of residents reported being involved in at least one robotic procedure, and $54 \%$ of these residents reported this experience by their third year of residency (Fig. 1). Experience with RAS was further broken down into participation role (observer, assistant or console operator) and training year (Fig. 2). Most residents reported involvement in fewer than five RAS cases in any one role, although a very small number reported console experience in more than 40 cases.

Residents were asked to rate their perceived knowledge of, and interest in, RAS. Sixty percent rated their interest in RAS as "medium." Of the smaller proportion who rated their interest in RAS as "low," 83\% had not been involved in a single RAS case. Interest in RAS tended to be rated higher by residents in programs that had a robot, with $38 \%$ of these residents reporting "high" interest compared to only $11 \%$ of residents at programs without a robot. Most residents (60\%) ranked their knowledge of RAS as "low." Furthermore, residents of programs without robots were about twice as likely to report "low" knowledge of RAS (89\% vs. 44\%).

Several survey questions assessed resident perception towards the feasibility, legitimacy and future of RAS (Table 2). There was mixed opinion regarding the feasibility of RAS in the current model of Canadian health care; most residents did not feel RAS was superior to existing surgical modalities. Despite this, residents felt strongly (92\%) that the prevalence of RAS will increase. It is interesting to note that $42 \%$ of residents in programs with a robot felt RAS was feasible in the Canadian system versus just $6 \%$ among residents in programs without a robot. Moreover, $44 \%$ of residents in programs with a robot felt RAS would become the new "gold standard" for certain surgical procedures compared to just $17 \%$ of residents in programs without robots. These two apparent differences in perception were mirrored between residents voicing high interest in RAS and those reporting low interest.

The attitudes of residents with respect to embracing RAS in their training and future career goals were also assessed

\begin{tabular}{|c|c|}
\hline & No. respondents (\%) \\
\hline \multicolumn{2}{|l|}{ Sex } \\
\hline Male & $42(84 \%)$ \\
\hline Female & $8(16 \%)$ \\
\hline \multicolumn{2}{|c|}{ Year of Training } \\
\hline 1 & $15(30 \%)$ \\
\hline 2 & $9(18 \%)$ \\
\hline 3 & $11(22 \%)$ \\
\hline 4 & $11(22 \%)$ \\
\hline 5 & $4(8 \%)$ \\
\hline \multicolumn{2}{|l|}{ Age } \\
\hline $20-25$ & $0(0 \%)$ \\
\hline $26-30$ & $41(82 \%)$ \\
\hline $31-35$ & $8(16 \%)$ \\
\hline $36-40$ & $0(0 \%)$ \\
\hline$>40$ & $1(2 \%)$ \\
\hline \multicolumn{2}{|c|}{ da Vinci system in program } \\
\hline Yes & $32(64 \%)$ \\
\hline No & $18(36 \%)$ \\
\hline
\end{tabular}

(Table 3). In general, residents did not feel that programs should expand their emphasis on RAS during residency. Those residents from programs with robots were split equally on this issue, whereas just $11 \%$ of residents from programs without a robot felt emphasis on RAS should be expanded. Of those respondents expressing high interest in RAS, almost $80 \%$ also reported interest in pursuing fellowship training in RAS.

In the final portion of the survey, residents were divided into two groups according to the presence or absence of a robot in their training program. They then answered several questions on how the presence or absence of a robot affected their training (Fig. 3). Most (44\%) residents in programs without a robot felt that it did not affect their training. In contrast, $68 \%$ of residents training in programs with a robot felt that its presence had a detrimental effect on training. This was felt strongest by fourth-year residents, $89 \%$ of whom felt the robot was detrimental to their training. In spite of this sentiment, $87 \%$ of residents with a robot in their program desired more console time (Fig. 4). Finally, those residents with a robot in their program were asked to rate their perceived level of access to the robot during RAS cases (Fig. 5). Most of these residents reported having little access to the robot in either assistant or operator roles.

\section{Discussion}

Surgeons who are trained in certain procedures during residency are more likely to perform these procedures in future practice.$^{13}$ Extrapolating this point to the resident experience with RAS (while recognizing unique features of RAS, such as cost and resource scarcity), one may make important 


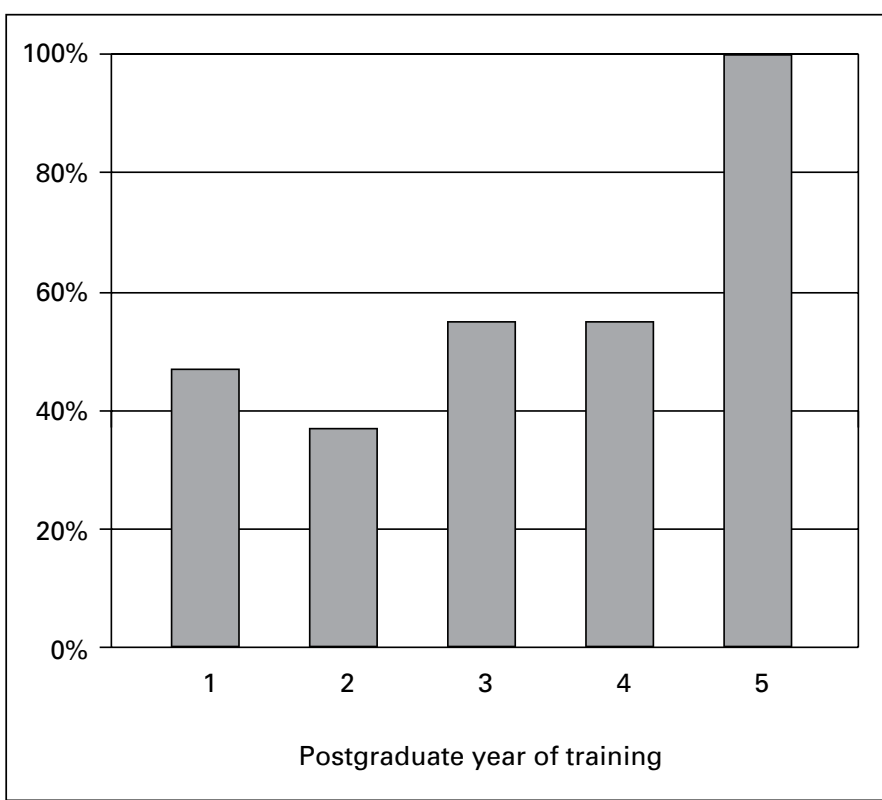

Fig. 1. Percentage of residents who have been involved in at least one roboticassisted surgery case according to year of training.

inferences about the future landscape of urologic practice in Canada. In this survey, more than half of residents reported being involved in at least one RAS procedure. Residents who reported access to RAS were more likely to report interest in pursuing fellowship training in RAS, and felt more likely to use RAS in the future. One recent study reported that between 2007 and 2008, the proportion of chief residents at Canadian urology training programs who had access to RAS rose from $4 \%$ to $36 \%$, with $39 \%$ of chief residents planning to do robotic surgery after residency. ${ }^{14}$ In the present study, of the residents who expressed low interest in RAS, over $80 \%$ also denied exposure to RAS and stated their little knowledge of RAS. It is not known what, if any, relationship exists between exposure to RAS and these other factors, but it will be interesting to follow this as exposure to RAS continues to increase.

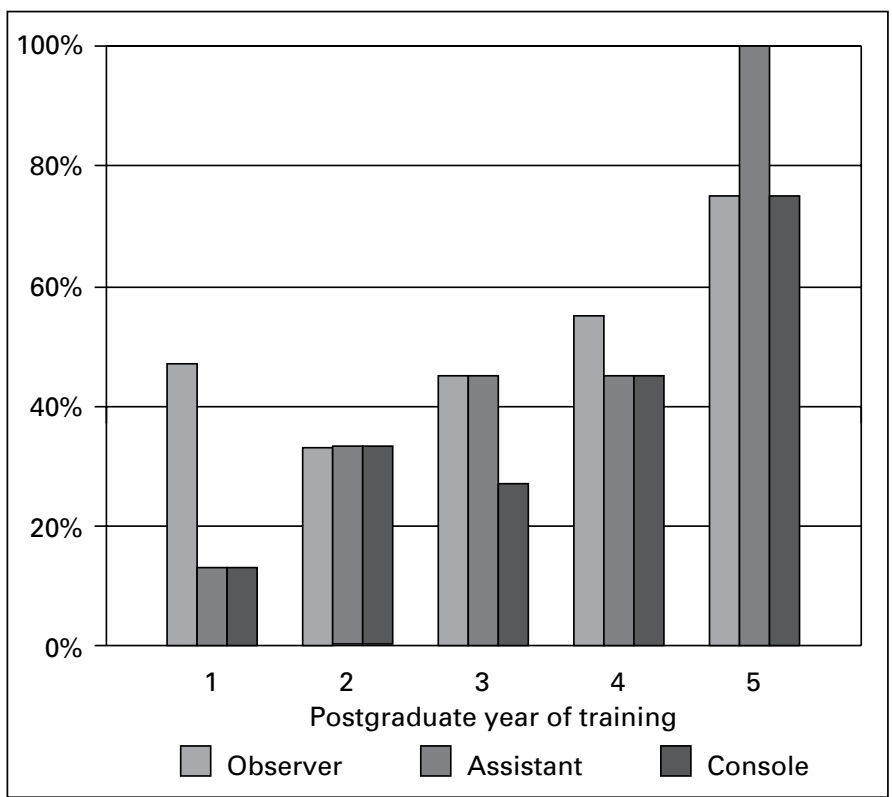

Fig. 2. Resident experience with robotic-assisted surgery according to role of involvement.

Several studies have attempted to quantify the prevalence of RAS in American training institutions. ${ }^{10-12}$ The most recent of these, which surveyed both urology residents and program directors in 2005, reported that urological RAS was being performed at $54 \%$ of institutions. This has no doubt increased significantly in the interim, and suggests Canadian urology residents lack the same level of access to RAS when compared to their American counterparts. In 2008, 75\% of da Vinci Surgical Systems in the world were located in the United States, compared to less than 1\% (nine systems) in Canada. ${ }^{15}$ Although most of the robotic systems in Canada are purchased via philanthropic foundations and private support, many (including most residents in this study) feel that RAS will continue to grow. In one multinational survey, ${ }^{4}$ $50 \%$ of surgeons felt the cost of the da Vinci Surgical System was the limiting factor in developing a RAS program at their

Table 2: Resident views on the future of robotic-assisted surgery

\section{What do you feel will happen to the prevalence of robotic-assisted surgery?}

Increase (92\%)

Decrease (2\%)

Do you feel robotic-assisted surgery will fulfill an increasingly important role in urology?

Yes $(59 \%) \quad$ No $(10 \%)$

Do you feel the use of robotic-assisted surgery is feasible within the Canadian health care system?

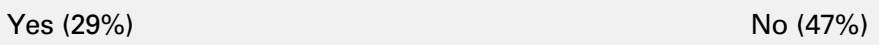

Do you feel robotic-assisted surgery is superior to traditional open surgical techniques?

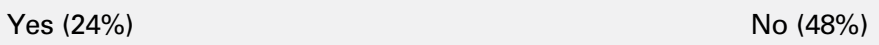

Do you feel robotic-assisted surgery is superior to laparoscopic surgical techniques?

$\begin{array}{lll}\text { Yes }(36 \%) & \text { No }(44 \%) & \text { Unsure (20\%) }\end{array}$

Do you feel robotic-assisted surgery will become the new gold standard for certain surgical procedures in urology?

Yes (34\%)
Remain unchanged (6\%)

Unsure (31\%)

Unsure (25\%)

Unsure (28\%)

Unsure (34\%) 


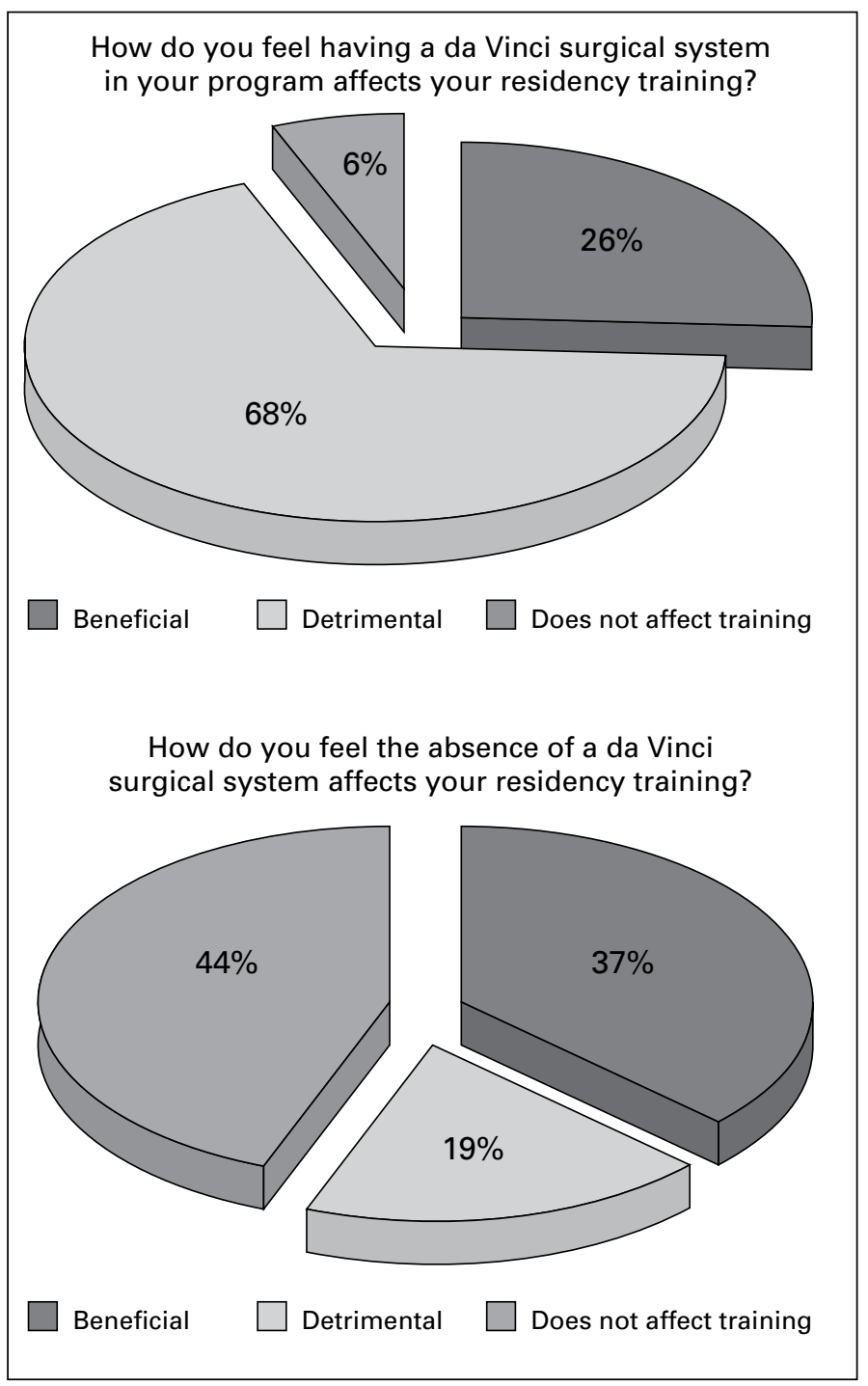

Fig. 3. Responses of residents training in programs with a robot (left) and residents in programs without a robot (right) when asked how either the presence or absence of a robot in their program affects their training.

institution. In Canada, the per-case cost of RAS is likely also a significant barrier. For example, the institutional cost of open radical prostatectomy is about $\$ 450$ per case, ${ }^{15}$ whereas that of a robotic-assisted laparoscopic prostatectomy (RALP) is $\$ 4800$. This estimate, however, does not account for the reduced costs realized as a result of shorter hospitalization and diminished perioperative morbidity suggested by proponents of RAS. Sensitivity to costs within our publicly funded health system may explain why nearly half of Canadian urology residents did not feel RAS was feasible within the Canadian health care model. Despite this, there seems a significant divide amongst residents on this issue; $42 \%$ of those in programs with a robot felt RAS was feasible in Canada, compared to just $6 \%$ of residents in programs without a robot.

Though the feasibility of RAS in Canada is debated, there

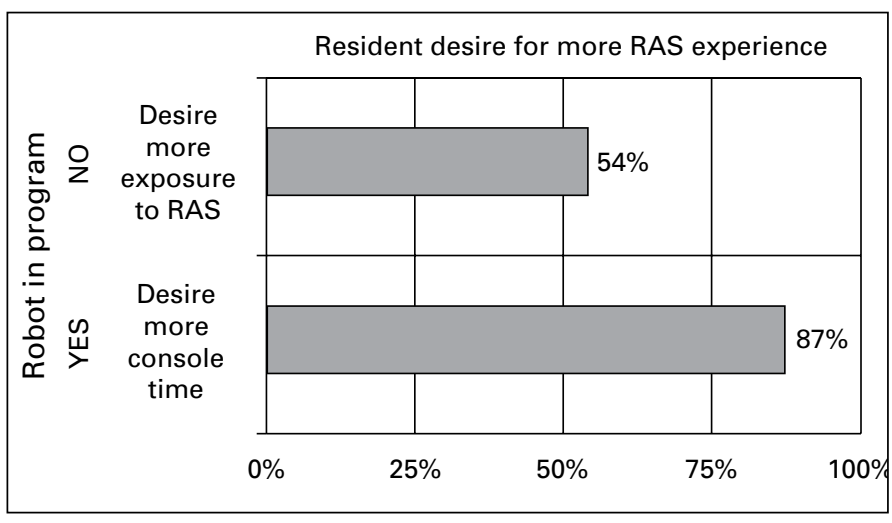

Fig. 4. Resident desire for more robotic experience according to whether or not their program has a robot. Response from residents in programs with no robot (top) vs. response of residents from programs with a robot (bottom) when asked if they desire more console time. RAS: robotic-assisted surgery.

seems to be emerging agreement regarding its future. In 2006 , Duchene and colleagues reported that $74 \%$ of residents and program directors in the United States felt that the application of RAS would increase. ${ }^{10}$ In addition, the authors found that $78 \%$ of these residents and surgeons felt it was either beneficial or essential to have training in RAS. In our study, $92 \%$ of residents felt that the application of RAS will increase. It is interesting that just $34 \%$ of residents in our study felt RAS would become the new gold standard for certain procedures; in a study by Guru and colleagues, $61 \%$ of respondents felt that RAS is already the gold standard. ${ }^{4}$ As the Guru study was a multinational survey of residents and surgeons ( $62 \%$ of whom denied having any console time during RAS), it is difficult to determine the cause of this opinion. It seems, however, that Canadian urology residents are more reserved in considering the superiority of RAS compared to traditional open or laparoscopic modalities.

As already demonstrated, there appears to be several significant differences in opinion when comparing residents in programs with robots to those without. With respect to the training environment, most residents in programs with a robot view its presence as detrimental to training; an even greater majority of residents in programs without a robot view its absence as either beneficial or inconsequential. Somewhat contradictory to this sentiment, however, is the expressed desire among most residents for more exposure to RAS. In the past decade, there has been a dramatic shift from open surgery to minimally invasive surgery. There is also some concern about the development of proficient surgical skills in light of the reduced exposure to open cases during residency. ${ }^{16}$ Unfortunately, there is a paucity of data quantifying how this shift has affected resident case volume. One survey of gynecology residents found a significant decrease in the number of cases completed in the surgeon role following introduction of RAS in their program. While this change was quite small, the impact on training was perceived as heavily negative. ${ }^{5}$ Canadian urology residents may ask this 


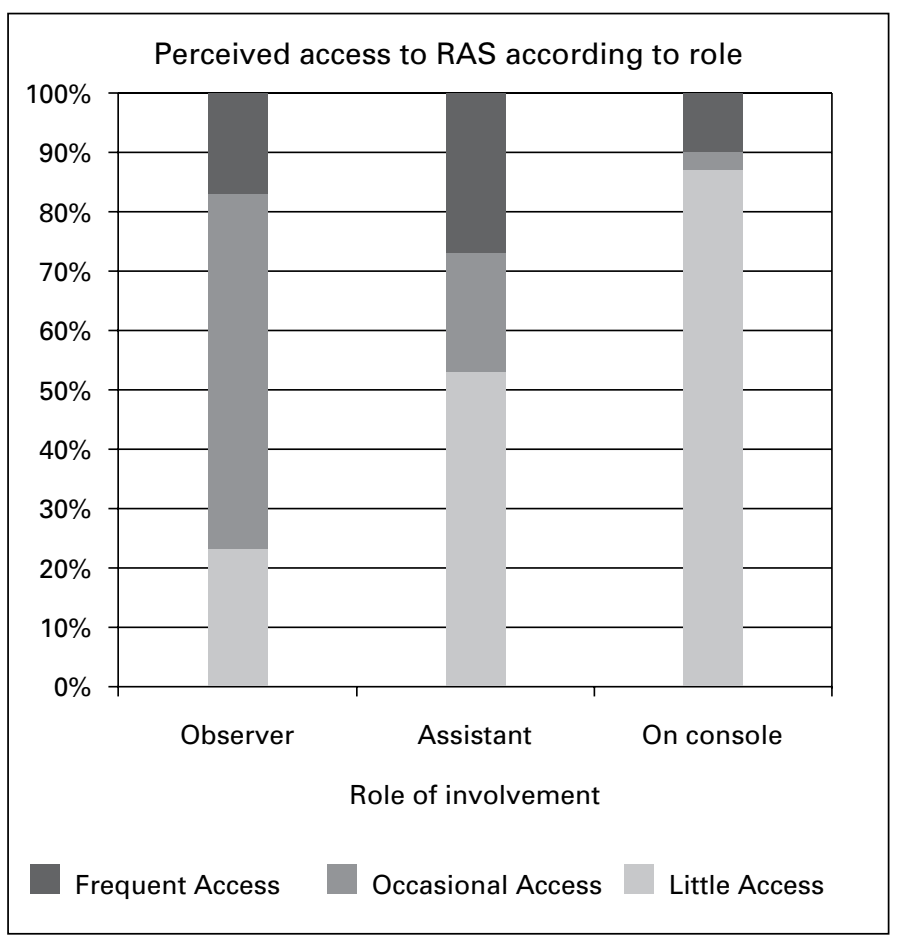

Fig. 5. Perceived resident access to robot according to role of involvement during robotic-assisted surgery. RAS: robotic-assisted surgery.

same question of RAS, and this perception that their volume of experience is being reduced may well be where the less favourable views towards RAS derive. Given that $86 \%$ of residents in programs with a robot report "little" access to the console, RAS may be viewed as detracting from valuable hands-on surgical experience even when the resident is involved in an assistant role.

The present survey demonstrates that residents are most often involved in observer and assistant roles during RAS cases, the value of which requires examination. Significant experiential differences exist pertaining to these roles when comparing RAS to open surgery. Some argue that observing and assisting during RAS cases help residents learn the procedure and develop 3-dimensional mental processing useful for laparoscopic and robotic surgery. While this may be true initially, it is well-documented that benefits from such experiences are limited to the first few occasions, after which the educational returns become increasingly small. ${ }^{12}$ It should be no surprise that a senior resident relegated to the repeated duty of holding a retractor as bedside assist during RAS cases would view this as a detriment to his or her training. Residents and attending staff are known to have significant differences in their perception of resident involvement during surgical cases. ${ }^{17}$ This implies that staff may view the resident learning experience quite differently than residents themselves, underscoring the utility of resident feedback. The relative novelty of RAS in Canadian training institutions makes this all the more important.

One may draw parallels between the controversies raised with the recent emergence of RAS and those raised with the introduction of laparoscopy in the late 1980s and early 1990s. Like laparoscopy, RAS is another milestone in surgical evolution, and, as was seen with laparoscopy, opinions will remain dynamic regarding RAS until our experience with it matures and we are able to analyze it more objectively. In this context, the negative impact that residents perceive RAS to have on training may more aptly reflect the early stage of acceptance of RAS in Canadian training programs. Simulation is probably the best method to disseminate training and expertise in the application of this new technology, but time and cost are major barriers. One may expect the resident opinion in programs with a robot to become more favourable as the attending urologists become more experienced with RAS and are better able to integrate residents more frequently and with greater roles of responsibility. By the same token, residents in programs without a robot may begin to view its absence more negatively. This may have important implications on resident recruitment. It is worth noting, however, that there are presently no Royal College resident training requirements pertaining to RAS. At a time when there is considerable "curriculum compression" in residency training, it seems unlikely that this will change in the near future. Notwithstanding this, the equal split between programs in this survey that have a robot and those that do not, makes the issue of how RAS affects resident training a timely one and worthy of further investigation.

There are several limitations of this study. The survey was not translated into French, which is perhaps the most significant limitation of this study. The francophone resident pool comprises a significant proportion of Canadian urology residents and these views and experiences have not been represented. In addition, a significant number of residents chose not to participate in this survey, and it may be that those less interested in RAS were also less likely to respond. Most respondents were junior residents, while residents in

\section{Table 3: Resident views on robotic-assisted surgery in training and future practice}

Do you feel your residency program should increase its emphasis on robotic-assisted surgery?

$\begin{array}{lll}\text { Yes }(33 \%) & \text { No }(49 \%) & \text { Unsure (18\%) }\end{array}$

Do you feel you will use robotic-assisted surgery in your career once you have completed residency?

$\begin{array}{lll}\text { Yes }(42 \%) & \text { No }(28 \%) & \text { Unsure }(30 \%)\end{array}$

Are you interested in pursuing fellowship training in robotic-assisted surgery

Yes (40\%) No (38\%)

Unsure (22\%) 
their final year of training were underrepresented, potentially adding bias to the results. It may be, however, that since robotics is an emerging technology, senior residents are in fact less familiar with this technology, and also less interested in adopting it at the end of their training (as it is not part of the Royal College objectives). By this logic, it would be most appropriate to focus on junior residents whose training will be most affected by this technology. Previous studies have raised the concern that Internet surveys may deter those who do not regularly check email or go online, but this is now likely an outdated notion given the ubiquity of the Internet and the necessity of email among residents. The non-validated questionnaire used in this study is another possible limitation.

\section{Conclusion}

The resident experience with respect to RAS is mixed. Overall, residents view RAS as an expanding field with a potentially negative impact on their present training, although they appear to desire more experience in RAS. Optimizing the resident training environment where RAS is used may include simple measures as implementation of a basic RAS curriculum, more access to the console and further investigation to quantify the impact of RAS on open and laparoscopic surgical case volume. Resident exposure and perceptions are likely to change in the coming years as staff progress past the learning stage facilitating broader resident experience. We plan to follow this over the next four years with further inquiry into resident access to surgical simulators, presence of RAS training curriculum and site-specific volume of RAS procedures.

Competing interests: None declared.

This paper has been peer-reviewed.

\section{References}

1. Davies BL, Hibberd RD, Coptcoat MJ, et al. A surgeon robot prostatectomy-a laboratory evaluation. J Med Eng Technol 1989;13:273-7. http://dx.doi.org/10.3109/03091908909016201

2. Thiel DD, Winfield HN. Robotics in urology: past, present, and future. J Endourol 2008;22:825-30. http://dx.doi.org/10.1089/end.2007.9830

3. Chin JL, Luke PP, Pautler SE. Initial experience with robotic-assisted laparoscopic radical prostatectomy in the Canadian health care system. Can Urol Assoc J 2007;1:97-101.

4. Guru KA, Hussain $A$, Chandrasekhar $R$, et al. Current status of robot-assisted surgery in urology: a multinational survey of 297 urologic surgeons. Can J Urol 2009;16:4736-41; discussion 4741.

5. Green I, Sokol A. Robotics in residency training: the evolution of technology and surgical education. I Minim Invasive Gynecol 2008; 15:93S-94S.

6. Pugh CM, DaRosa DA, Glenn D, et al. A comparison of faculty and resident perception of resident learning needs in the operating room. J Surg Educ 2007;64:250-5. http://dx.doi.org/10.1016/i. jsurg.2007.07.007

7. $K_{0} C Y$, Escarce JJ, Baker L, et al. Predictors of surgery resident satisfaction with teaching by attendings: a national survey. Ann Surg 2005;241:373-80. http://dx.doi.org/10.1097/01. sla.0000150257.04889.70

8. Ratnapalan S, Ito S. Pediatric resident education and needs assessment in clinical pharmacology. Can J Clin Pharmacol 2004;11:e150-5.

9. Nadol JB Jr. Training the physician-scholar in otolaryngology-head and neck surgery. Otolaryngol Head Neck Surg 1999;121:214-9. http://dx.doi.org/10.1016/S0194-5998(99)70174-9

10. Duchene DA, Moinzadeh A, Gill IS, et al. Survey of residency training in laparoscopic and robotic surgery. J Urol 2006;176:2158-66; discussion 2167. http://dx.doi.org/10.1016/i.juro.2006.07.035

11. Patel YR, Donias HW, Boyd DW, et al. Are you ready to become a robo-surgeon? Am Surg 2003;69:599603.

12. Donias HW, Karamanoukian RL, Glick PL, et al. Survey of resident training in robotic surgery. Am Surg 2002;68:177-81

13. Shay BF, Thomas R, Monga M. Urology practice patterns after residency training in laparoscopy. J Endourol 2002;16:251-6. http://dx.doi.org/10.1089/089277902753752232

14. Preston MA, Blew BD, Breau RH, et al. Survey of senior resident training in urologic laparoscopy, robotics and endourology surgery in Canada. Can Urol Assoc J 2010;4:42-6.

15. Estey EP. Robotic prostatectomy: The new standard of care or a marketing success? Can Urol Assoc J 2009:3:488-90.

16. MacNeily AE. The training of Canadian urology residents: Whither open surgery? Can Urol Assoc J 2010;4:47-8.

17. Yap SA, DeLair SM, Tanaka ST, et al. Current perceptions of resident training in laparoscopic nephrectomy. Urology 2009;73:1067-71. http://dx.doi.org/10.1016/i.urology.2008.08.520

Correspondence: Dr. Michael Robinson, Department of Urologic Sciences, Faculty of Medicine, University of British Columbia, Level 6, 2775 Laurel St., Vancouver, BC V5Z 1M9; fax: 604-8754637; meric@interchange.ubc.ca 


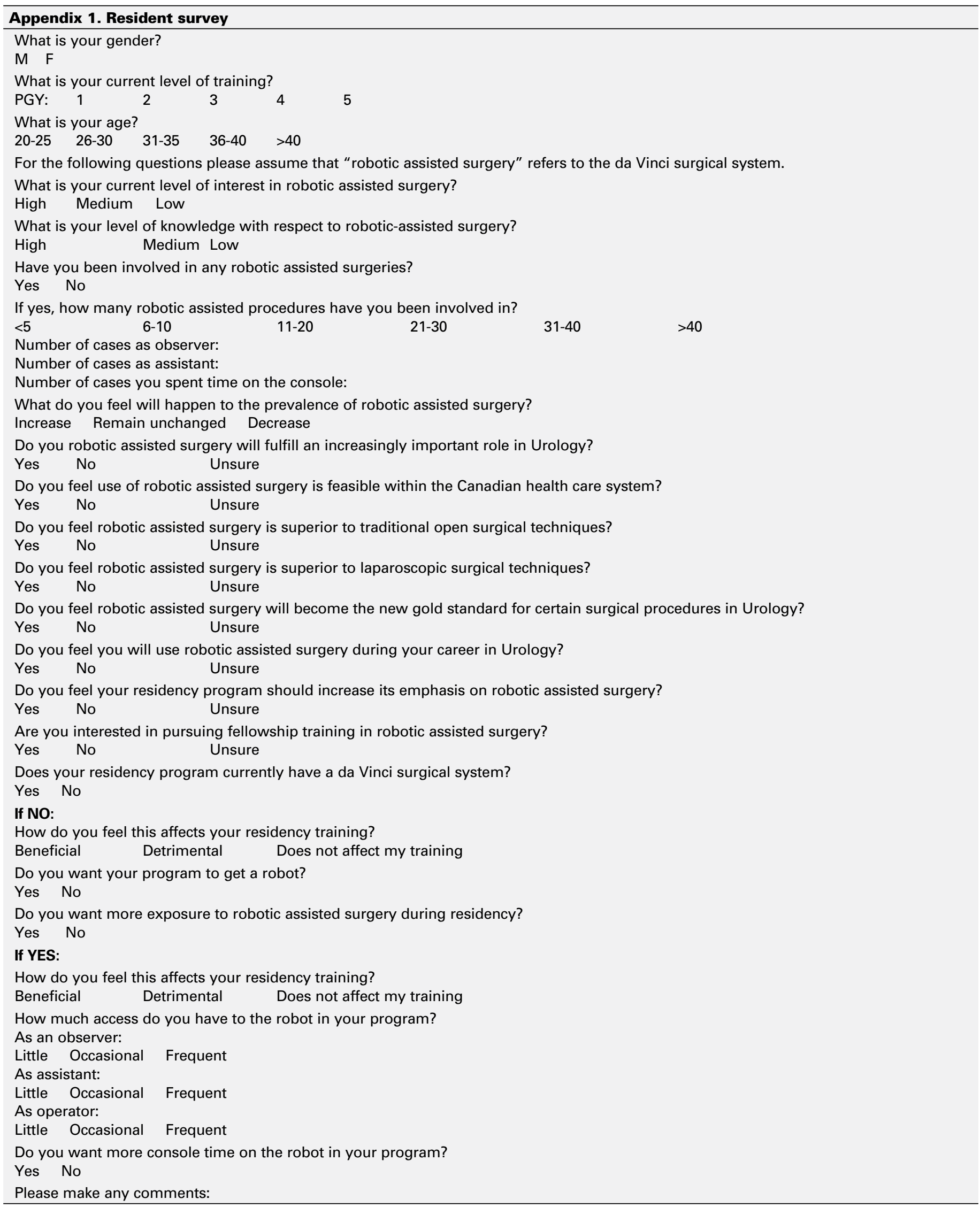




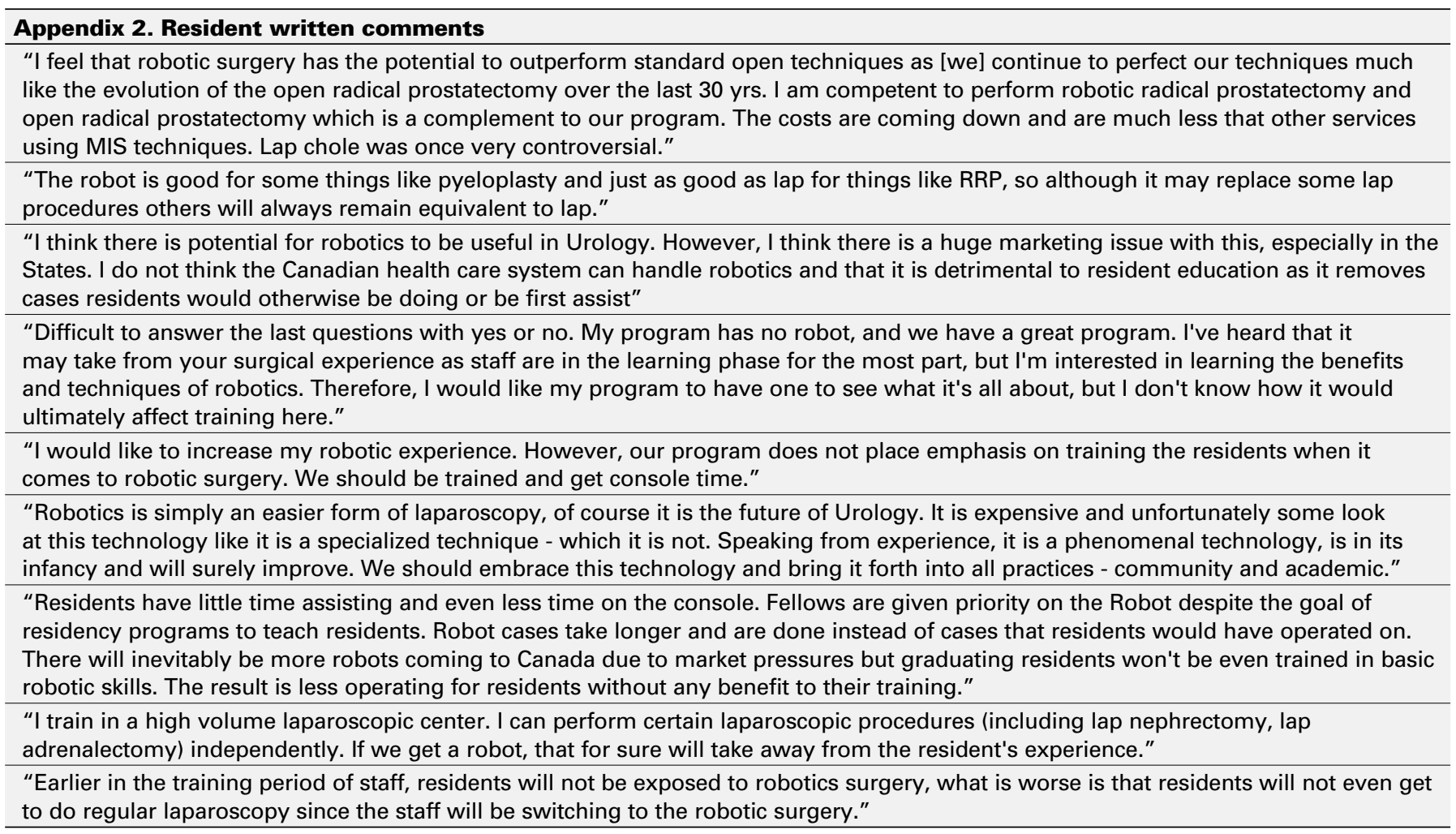

